\title{
ON THE CRITICAL CONDITIONS FOR AN ASSEMBLY OF INTERACTING THERMONS
}

\author{
B. F. GRAY ${ }^{1}$ \\ (Received 20 November, 1998; revised 20 April, 1999)
}

\begin{abstract}
The interaction of a number of self-heating bodies depositing heat into a common finite heat bath and thereby influencing each other is a problem of great practical importance in many areas including storage and transport of self-heating materials, drums of chemicals, foodstuffs etc. Critical conditions for the complete assembly of interacting heat producers (thermons) are derived under various assumptions and modes of ignition are identified. These include cooperative modes as well as modes which are simply perturbations of ignition for single thermons.
\end{abstract}

\section{Introduction}

Self heating is a problem of both immense practical and industrial interest and also academic interest. It gives rise to many interesting nonlinear equations involving the ubiquitous Arrhenius function $\exp (-1 / u)$ where $u$ is a dimensionless temperature which is bounded away from zero. An immense amount of work has been carried out on individual self-heating bodies placed in fixed ambient temperatures with various forms of time independent boundary conditions. Bowes' book [2] gives a fairly comprehensive account of a large part of this work. A small amount of work has been done on time dependent boundary conditions of an explicitly given functional form, for example [6], but no attention seems to have been paid to a situation of very great practical importance and frequent occurrence, that is, a number of self-heating bodies (thermons) discharging heat into a common atmosphere of finite thermal capacity $([7,8])$. Practical examples of this abound - storage of charcoal containers in a ship's hold (see [2]) and the transport of any self-heating material in kegs, drums or cartons, inside a container, which itself may be on a truck or inside the hold of a ship. The essential point of interest is that an extra thermal resistance is inserted between

${ }^{1}$ School of Chemistry, Macquarie University, Sydney, Australia.

(C) Australian Mathematical Society 2001, Serial-fee code 0334-2700/01 


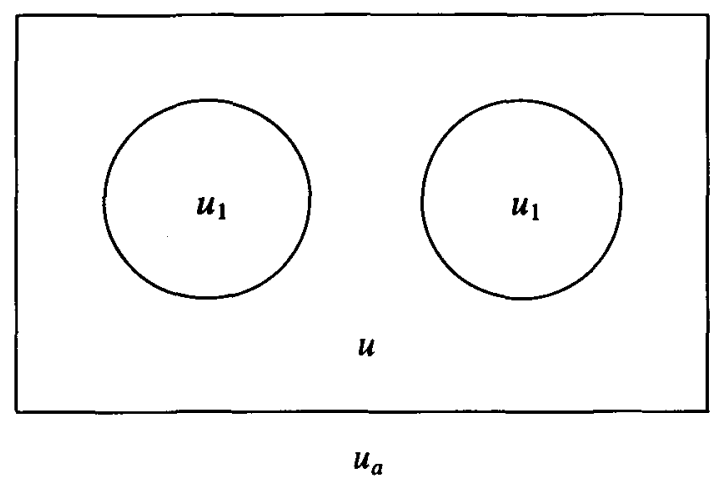

FIGURE 1. Schematic diagram of a possible configuration.

the reactive self-heating material and the infinite thermal capacity thermostat (the outside atmosphere) implied by the time independent boundary conditions. Cartons of oxidisable food products (such as noodles) shrink-wrapped onto $1.4 \mathrm{~m}^{3}$ pallets can interact significantly in this way and Clancey [3] has drawn attention to the many disastrous fires suspected of being caused by calcium hypochlorite (pool chlorine) shipped in drums packed in $20 \mathrm{ft}$ containers which are themselves stacked inside ships' holds at hold temperatures which may be as high as $40^{\circ} \mathrm{C}$.

The generic mathematical problem posed is an interesting and in complete generality, an intractable one. However tractable formulations which contain the essence of the problem and give semi-quantitative results can be found.

Reference to Figure 1 shows that in general, there are great geometrical complications. This shows a system in plan-it can be interpreted as showing a number of drums of circular cross section in a container shaped as a rectangular brick-this would be a common practical case. Here $T_{a}$ is a fixed ambient temperature-the outer atmosphere, and $T$ is the (generally time dependent) temperature of the air inside the container-this is the medium through which the self-heating bodies interact. The self-heating bodies, each with internal temperature distribution $T_{i}(\mathbf{r}, t)$ will usually be identical (we will assume this) and will usually have identical initial conditions. Clearly a very important parameter in the problem will be the heat transfer coefficient of the outer container wall $W$. In the limit as this quantity $\rightarrow \infty$ (or as the size of the outer container $\rightarrow \infty$ ) the interaction between the drums $\rightarrow 0$ and they behave as single bodies placed in a heat bath at constant temperature $T_{a}$. Our task is to investigate the dependence of the initial condition for ignition (saddle-node bifurcation locus) on the heat transfer through the outer container to the (assumed) atmospheric heat bath and on the number of bodies inside the container. We commence this task by examining some approximations which result in tractable, but still meaningful, formulations of the general problem. 


\section{The "well-stirred" formulation}

It has proved a very useful procedure in thermal ignition theory, when investigating a new problem such as this one, to firstly remove geometry from consideration by following the procedure originally formulated in order to study the dynamics of igniting systems, that is, as an initial value problem. In this section we will follow this procedure and view the problems as a dynamical system.

We consider $n$ identical self-heating bodies, not in direct contact with each other, placed inside a container as in Figure 1. The bodies are not in direct contact with the container wall. The container is placed in a constant temperature heat bath, temperature $T_{a}$. We can write an energy conservation equation for each body in the form

$$
c \rho v \frac{d T_{i}}{d t}=v Q Z \exp \left(-\frac{E}{R T_{i}}\right)-\chi s\left(T-T_{i}\right), \quad T_{i}(0)=T_{i}^{0}, \quad i=1, \ldots, n,
$$

where $T_{i}$ is the (spatially constant) temperature of each body, $v$ is the volume of each body, $c$ is the heat capacity, $\rho$ is the density, $Q$ is the heat of reaction, $Z$ is the preexponential factor and $E$ the activation energy of the reaction. Here $R$ is the universal gas constant and $s$ is the surface area of each body. The heat transfer coefficient from the surface of the solid body to the air inside the container is given by $\chi$. We are assuming here that the fuel concentration remains at its initial value on the time scale under consideration. This is discussed in detail in [5]. It is worth noting at this point that this number is much more sensitive to the physical nature of the solid surface than it is to the nature, and thermal conductivity, of the material itself. For a smooth surface heat transfer from the solid to the gas phase the boundary layer is important. For heat transfer through the container wall, two such transfers must occur, that is, air $\rightarrow$ solid followed by solid $\rightarrow$ air. If we assume the air inside the container is as well stirred as that in the heat bath at $T_{a}$, then the outer heat transfer coefficient will be approximately $0.5 \chi$, since overall the process involves two air/solid processes in series.

The energy conservation for the container can be written down with the same assumptions:

$$
C P V \frac{d T}{d t}=\chi s \sum_{i=1}^{n}\left(T_{i}-T\right)-\psi S\left(T-T_{a}\right),
$$

where $C, P$ and $V$ now refer to the air inside the container. We denote by $\psi$ the heat transfer coefficient for the series heat transfer processes container air $\rightarrow$ container wall; container wall $\rightarrow$ ambient air and this should be approximately $0.5 \chi$. The surface area of the container (walls assumed thin) is given by $S$. If $V_{0}$ is the volume of the container, $V=V_{0}-n v$. 
It is convenient to make these equations dimensionless and they can be written in the form

$$
\begin{array}{rlrl}
\frac{d u_{i}}{d \tau} & =\exp \left(-1 / u_{i}\right)-l\left(u_{i}-u\right), & u_{1}(0)=u_{i}^{0}, & i=1, \ldots, n, \\
\alpha \frac{d u}{d \tau} & =l \sum_{i=1}^{n}\left(u_{i}-u\right)-\beta l\left(u-u_{a}\right), & u(0)=u^{0},
\end{array}
$$

where

$$
\begin{array}{lll}
u_{i}=R T_{i} / E, & \tau=Z R E Q / c \rho E, \quad l=\chi s E / v Q R Z, & u=R T / E, \\
u_{a}=R T_{a} / E, & \alpha=C P V / c \rho v=C P\left(V_{0}-n v\right) / c \rho v, & \beta=\psi S / \chi s .
\end{array}
$$

It is worth noting here that the requirement $V_{0}-v>0$ bounds $n$, assuming $V_{0}$ and $v$ given, there is no relationship between $S, s$ and $n$. In many realistic cases $n s \gg S$.

The dimensionality of the problem can be reduced to two for the physically important case $u_{i}(0)=u_{i}=u_{i}^{0}$ since this implies $u_{i}(\tau)=u_{j}(\tau), \tau>0$ for all $i, j$, that is, all the drums behave identically. Many physically interesting cases arise where this is not so, for example where one drum is initially hotter than the rest, the question as to how much hotter it can be before it pushes the assembly over the ignition watershed is an important one which will be addressed in a later paper. Here we will restrict ourselves to the most important practical special case of identical initial conditions for each self-heating body. This being two-dimensional is also of high pedagogical value. Equations ( 3 ) and (4) become

$$
\begin{aligned}
\frac{d u_{1}}{d \tau} & =\exp \left(-1 / u_{1}\right)-l\left(u_{1}-u\right), \\
\alpha \frac{d u}{d \tau} & =\ln \left(u_{1}-u\right)-l \beta\left(u-u_{a}\right) .
\end{aligned}
$$

\section{Special case - A two-dimensional system}

Here we examine (5) using standard techniques. We just examine the singularities satisfying

$$
\begin{aligned}
\exp \left(-1 / u_{1}\right)-l\left(u_{1}-u\right) & =0 \\
\ln u_{1}-l(n+\beta) u+l \beta u_{a} & =0,
\end{aligned}
$$

from which $u$ can be eliminated giving the bifurcation problem

$$
\exp \left(-1 / u_{1}\right)-\frac{l\left(u_{1}-u_{a}\right)}{(1+n / \beta)}=0 .
$$

This is a familiar equation with the interaction represented by the term $(1+n / \beta)^{-1}$ modifying the loss coefficient. As $\beta \rightarrow \infty$ the interaction tends to zero and the 
familiar problem is retained. The locus of saddle node bifurcations is obtained in the usual way by solving ( 7 ) along with $\operatorname{det} \mathbf{J}=0$, where $\mathbf{J}$ is the Jacobian matrix. This provides us with the condition

$$
\frac{\exp \left(-1 / u_{1}\right)}{u_{1}^{2}}-\frac{l}{(1+n / \beta)}=0 .
$$

Equations (7) and (8) can be solved for $u_{1}$ to give the result at criticality

$$
u_{1}=(1 / 2) \pm\left(1-4 u_{a}\right)^{1 / 2} / 2 \text {. }
$$

For most cases of practical interest $u_{a} \ll 1$. Hence

$$
\begin{aligned}
& u_{1}^{+} \simeq 1-u_{a}-u_{a}^{2}, \\
& u_{1}^{-} \simeq u_{a}+u_{a}^{2},
\end{aligned}
$$

$u_{1}^{-}$referring to the ignition bifurcation in which we are most interested. Substitution in (7) gives us an approximation for the ignition locus in parameter space as

$$
l=\left(1+\frac{n}{\beta}\right) \frac{\exp \left[-1 / u_{a}\left(1+u_{a}\right)\right]}{u_{a}^{2}} .
$$

A more interesting form of this equation is

$$
n=\beta\left\{l u_{a}^{2} \exp \left[1 / u_{a}\left(1+u_{a}\right)\right]-1\right\} .
$$

If the number of self-heating bodies exceeds this figure then ignition will occur-it is the maximum safe number of self-heating bodies storable under the conditions defined by the parameters $l, \beta$ and $u_{a}$.

Examination of the Jacobian matrix for the system (5) shows that Hopf bifurcations are not possible. It is conjectured that periodic solutions of (5) do not exist.

3.1. $\alpha \rightarrow 0^{+}$Asymptotics involving extreme values of the parameter $\alpha$ give some interesting insights when two clearly distinguished time scales occur. Recalling the definition of $\alpha=C P V / c \rho v$, if the reacting bodies are separated by air and they constitute either solid or liquid, then we can expect $\alpha \ll 1$. In this case we expect motion to occur on the slow subspace according to the equations

$$
\begin{aligned}
\frac{d u_{1}}{d \tau} & =\exp \left(-1 / u_{1}\right)-l\left(u_{1}-u\right), \\
u & =\frac{n}{(n+\beta)} u_{1}+\frac{\beta u_{a}}{n+\beta},
\end{aligned}
$$

which reduce to

$$
\frac{d u_{1}}{d \tau}=\exp \left(-1 / u_{1}\right)-\frac{l}{(1+n / \beta)}\left(u_{1}-u_{a}\right)
$$


Thus for small $\alpha$ and arbitrary initial conditions we expect rapid motion at constant $u_{1}$ onto the slow subspace followed by evolution according to (15). Physically the rapid variation of $u$ represents the adjustment of the air temperature inside the container to a value compatible with the initial temperature of the solid or liquid reacting material.

Numerical computation shows the existence of a large region in the $u_{1}-u$ plane such that initial conditions in that region lead to ignition for $n=40$ but not for $n=1$, for example. At first sight this is surprising since the ignition region in parameter space increases with $n$. This phenomenon occurs for relatively large $u$ and low $\beta$ and can easily be understood in physical terms. The energy contained in the warm or hot air inside the container raises the temperature of the solid reactive material on the short time scale. The greater the thermal capacity of this material (larger $n$ ) the smaller will be the resulting temperature rise in $u_{1}$ and the resulting value may well be on the lower side of the separatrix.

3.2. $\alpha \rightarrow \infty$ This case would represent bubbles or pores of reactive gas in a liquid or solid matrix, itself in a thermostated environment. It is convenient to rewrite (5) with $\epsilon=1 / \alpha \rightarrow 0^{+}$:

$$
\begin{aligned}
& \frac{d u_{1}}{d \tau}=\exp \left(-1 / u_{1}\right)-l\left(u_{1}-u\right), \\
& \frac{d u}{d \tau}=\epsilon l\left\{n\left(u_{1}-u\right)-\beta\left(u-u_{a}\right)\right\} .
\end{aligned}
$$

On the fast $(\tau)$ time scale, to zeroth order, we have to deal with the transparent problem

$$
\frac{d u_{1}^{(0)}}{d \tau}=\exp \left(-1 / u_{1}^{(0)}\right)-l\left[u_{1}^{(0)}-u(0)\right]
$$

Clearly if $u(0)$, which is now to be regarded as the (fixed) ambient temperature for the reacting gas, is above the critical value ignition will occur. The whole ignition process occurs on the fast time scale, interaction between the bubbles playing little or no role. A much more interesting case is where the initial value $u(0)$ is not sufficiently high to ignite a single bubble. We are interested in the existence of regions where "cooperative ignition" of the bubbles can occur. If we transform to the slow time $\tau^{\prime}=\epsilon \tau$ (16) becomes (on zeroth approximation $\epsilon \rightarrow 0^{+}$)

$$
\begin{gathered}
\exp \left(-1 / u_{1}^{(0)}\right)-l\left(u_{1}^{(0)}-u^{(0)}\right)=0, \\
\frac{d u^{(0)}}{d \tau^{\prime}}=l\left[n\left(u_{1}^{(0)}-u^{(0)}\right)-\beta\left(u^{(0)}-u_{a}\right)\right],
\end{gathered}
$$

where we are now assuming that $u_{1}^{(0)}$ is on the minimal solution branch of (18). After elimination of $u^{(0)}$ and some rearrangement we find that the slow motion is defined by 
the single equation

$$
\frac{\mathrm{d} u_{1}^{(0)}}{\mathrm{d} \tau^{\prime}}=\frac{l(n+\beta)\left[\exp \left(-1 / u_{1}^{(0)}\right)-l\left(u_{1}^{(0)}-u_{a}\right) /(1+n / \beta)\right]}{l-\exp \left(-1 / u_{1}^{(0)}\right) / u_{1}(0)^{2}}
$$

and the terms are such that this motion commences in a region of increasing $u_{1}^{(0)}$, both numerator and denominator being positive. This slow motion proceeds until the singularity in (20) is approached when ignition occurs and the approximation breaks down. Numerical simulation shows this interesting highly cooperative form of ignition very clearly. An initial rapid relaxation onto the slow manifold followed by prolonged development along it, finally terminating in ignition.

In physical terms this situation can be very misleading and very dangerous giving rise to extremely delayed ignition compared with the typical time scale for ignition of a single body, at ambient temperatures somewhat lower than expected for a single body.

\section{The general (unsymmetrical) case}

In the general problem as described by (3) and (4), if we do not assume equal initial conditions for all of the reacting bodies we obviously cannot achieve a reduction in dimensionality. However this increase in dimensionality raises very interesting questions of symmetry. We will restrict ourselves to a brief study of the singularities of the general system and consider the defining equations for them

$$
\begin{aligned}
& \exp \left(-1 / u_{i}\right)-l\left(u_{i}-u\right)=0, \quad i=1, \ldots, n, \\
& \sum_{i=1}^{n}\left(u_{i}-u\right)-\beta\left(u-u_{a}\right)=0
\end{aligned}
$$

The interesting point is that for any given value of $u$, each of the first $n$ equations of (21) will have either 1,2 or 3 solutions. The case of two solutions would be on a bifurcation locus so we will exclude that case for the present and concentrate on the case of three possible solutions. We would expect a series of steady states for the assembly in which each of the individual bodies could be in any one of the possible individual states ignoring stability considerations for the moment. Naively we might expect an assembly of $n$ bodies to exhibit $3^{n}$ steady states all told, with a range of stabilities. Given this possibility one then has to ask what the bifurcation diagram would look like, and indeed what would be an appropriate norm?

Here we merely attempt to throw some light on this question by looking at asymptotics for $\beta \rightarrow \infty$. Remembering our notation, $\epsilon=1 / \beta$, we assume for $\epsilon \rightarrow 0^{+}$,

$$
u_{i}=u_{i}^{(0)}+\epsilon f_{i}+O\left(\epsilon^{2}\right)
$$


when the $u_{i}^{(0)}$ are the known solutions for each noninteracting body, also known to possess three branches. Substitution of (22) into (21) gives, after the usual manipulation, the results

$$
\begin{aligned}
& u_{i} \simeq u_{i}^{(0)}+\epsilon l \frac{\sum_{j=1}^{n} u_{j}^{(0)}-n u_{a}}{l-\exp \left(-1 / u_{i}^{(0)}\right) / u_{i}^{(0)^{2}}}, \\
& u \simeq u^{(0)}+\epsilon\left(\sum_{j=1}^{n} u_{j}-n u_{a}\right) .
\end{aligned}
$$

Clearly if the asymptotics are valid, (23) shows that there is one to one correspondence between the assembly states with no interaction and those with weak interaction. In (23) we have to note that each of the functions $u_{j}^{(0)}$ on the right-hand side can in fact refer to any one of three branches. Stability questions remain to be answered. For example in the case of two bodies, one in the lower (individually stable) state and one in the upper (also individually stable) state, will the aggregate assembly be in a stable (doubly degenerate) state? This question appears related to questions of stability of spatially asymmetrical solutions of symmetrically posed problems discussed in a combustion [1] and general [4] context.

The differential equations for the $f_{1}(\tau)$, although linear, inevitably involve the unknown zeroth order solutions. In fact the equations are easily shown to be

$$
\frac{d f_{i}}{d \tau}=\left(\frac{\exp \left(-1 / u_{i}^{(0)}\right)}{u_{i}^{(0)^{2}}}-l\right) f_{i}+l \sum_{j=1}^{n}\left(u_{j}^{(0)}-u_{a}\right), \quad i=1, \ldots, n .
$$

For sufficiently large $\tau$ we know that $u_{i}^{(0)} \rightarrow \bar{u}_{i}^{(0)}$ where $\bar{u}_{i}^{(0)}$ is one of the stable steady solutions of the unperturbed assembly. This implies that

$$
\frac{\exp \left(-1 / u_{i}^{(0)^{2}}\right)}{u_{i}^{(0)^{2}}}-l \rightarrow-\left|\lambda_{i}\right|, \quad \tau \rightarrow \infty,
$$

where the $\left|\lambda_{i}\right|$ are constant. So for sufficiently large $\tau,(24)$ assumes the form

$$
\frac{d f_{i}}{d \tau} \simeq-\left|\lambda_{i}\right| f_{i}+l \mu
$$

where $\mu>0$ is a constant. Thus it appears likely that for large $\tau$

$$
u_{i} \rightarrow u_{i}^{(0)}+\frac{\epsilon l \mu}{\left|\lambda_{i}\right|}, \quad \tau \rightarrow \infty,
$$

provided $u_{i}^{(0)}$ is stable. This may not hold in higher approximations. The critical condition itself for the general case, that is, for a saddle-node bifurcation, is given by equations (21) plus the condition

$$
\text { Det } J=0 \text {, }
$$


which is readily shown to give the expression

$$
\frac{d}{d u}\left[\mathscr{R}(u)-\beta l\left(u-u_{a}\right)\right]=0,
$$

where $\mathscr{R}(u)$ is the total chemical heat generation rate in the system. The symmetry of $J$ rules out the possibility of Hopf bifurcations in this system.

\section{Some analytical solutions}

With truncation of the Arrhenius function to simple exponential form it is well known in the combustion literature, for example [2], that analytical solutions are known for the infinite slab and the infinite cylinder. For a fifth power law in place of the Arrhenius function an analytical solution is also known for the sphere and hollow sphere. Here we will illustrate the use of the Chambré [1] solution for the infinite cylinder in a context with interaction.

Let us consider $n$ infinite cylinders (pipes) in an infinite box (conduit) which itself is placed in a constant temperature thermostat.

If we use the traditional combustion dimensionless variables $\delta$ and $\theta$, the equation describing the reacting medium in each pipe is (we are now looking at steady spatially varying solutions)

with boundary conditions

$$
\frac{d^{2} \theta}{d \rho^{2}}+\frac{1}{\rho} \frac{d \theta}{d \rho}+\delta e^{\theta}=0
$$

$$
\begin{aligned}
\frac{d \theta}{d \rho}=0, & \rho=0, \\
\dot{\theta}=\theta_{1}, & \rho=1 .
\end{aligned}
$$

Here $\theta$ is defined with respect to the ambient temperature outside the conduit and $\theta_{1}$ is the spatially constant temperature in the dead space inside the conduit. We have used the radius of the pipes (identical) as the length scale giving

$$
\delta=\frac{Q Z \rho r^{2} E}{\kappa R T_{a}^{2}} \exp \left(-E / R T_{a}\right)
$$

where $Q, Z, E, T_{a}$ and $R$ are already defined and $\kappa$ is the thermal conductivity of the reacting material inside each pipe, and $r$ is the radius of each pipe.

The analytical solution inside each pipe is

$$
e^{\theta}=\frac{8 B}{\delta\left(1+B \rho^{2}\right)^{2}}
$$


where $B$ is a constant. If we apply the boundary condition at $\rho=1, \theta=\theta_{1}$, we get

$$
e^{\theta_{1}}=8 B /\left(\delta(1+B)^{2}\right)
$$

and the dimensionless heat flux from each pipe into the conduit is

$$
\left(\frac{d \theta}{d \rho}\right)_{\rho=1}=\frac{4 B}{1+B}
$$

The steady state equation for the conduit containing $n$ pipes and losing heat to the surrounding thermostat at $\theta=0$ is simply then

$$
\frac{4 B n}{1+B}=\mathscr{L} \theta_{1}
$$

where $\mathscr{L}$ is the dimensionless heat transfer coefficient through the conduit. In fact

$$
\mathscr{L}=\frac{S h}{2 \pi \kappa}
$$

where $S$ is the surface area per unit length of the conduit and $h$ is the heat transfer coefficient through the conduit wall. For a single pipe in a concentric circular conduit it is possible to write down an analytical solution for the case of finite thermal conductivity of the air space. It is a simple matter to show that the critical ambient temperature for this case is lower than the well-stirred case discussed here, which represents arbitrarily large thermal conductivity of the air space.

If we take exponentials of (37) and use (35) we obtain

$$
\delta=\frac{8 B}{(1+B)^{2}} \exp [-4 B n / \mathscr{L}(1+B)] .
$$

In the traditional way we define the critical value of $\delta$ by

$$
\frac{\partial \delta}{\partial B}=0 \text {. }
$$

This gives us the critical value of $B, B_{c}$ from

$$
\frac{\mathscr{L}}{n}=\frac{4 B_{c}}{\left(1-B_{c}\right)\left(1+B_{c}\right)}
$$

which gives

$$
B_{c}=\left(1+4 n^{2} / \mathscr{L}^{2}\right)^{1 / 2}-2 n / \mathscr{L} .
$$

It is most concise to regard (39) and (42) as a parametric definition of $\delta_{c}$ so that

$$
\delta_{c}=\frac{8 B_{c}}{\left(1+B_{c}\right)^{2}} \exp \left[-\frac{4 B_{c} n}{\mathscr{L}\left(1+B_{c}\right)}\right], \quad B_{c}=\left(1+\frac{4 n^{2}}{\mathscr{L}^{2}}\right)^{1 / 2}-\frac{2 n}{\mathscr{L}} .
$$


This tends to the well known Chambré solution for $\mathscr{L} \rightarrow \infty$, that is, $\delta_{c}=2, B_{c}=1$ and it is a trivial matter to obtain expansions for small $n / \mathscr{L}$. In fact to order $(n / \mathscr{L})$ we have

$$
\delta_{c} \simeq 2(1-2 n / \mathscr{L}) .
$$

It is interesting that criticality depends only on the ratio of $n / \mathscr{L}$ exactly.

Similar expressions can be obtained for the infinite slab and sphere without difficulty.

\section{Conclusions}

The interaction of self-heating bodies (thermons) can be treated both from the dynamical systems point of view and the stationary spatial distribution point of view, as can individual thermons. Applications are widespread such as in storage and shipping situations where drums or kegs of material are shipped inside containers which have generally poor heat transfer characteristics and cause significant interaction.

Also dense dust clouds in which the particles are porous may well exhibit significant interaction between the regions of reaction. Similarly granular materials may in some cases be more appropriately treated by the concept of interaction of thermons rather than use of a continuum model. The model also applies to vapour phase reaction in pores or bubbles in a liquid or solid medium, the interactions being particularly strong if the medium has a high thermal conductivity. In this case times to ignition can show unexpected dependence on parameter values.

Finally the treatment outlined here presents no obstacles to generalisation of the Arrhenius function to other temperature dependencies, and this is particularly important where complex chemistry is responsible for the self-heating.

\section{References}

[1] R. Aris, "Mathematical theory of diffusion and reaction in permeable catalysts", 1 (Oxford University Press, 1975), pp. 329-345.

[2] P. C. Bowes, Self heating: evaluating and controlling the hazards (H. M. Stationery Office, 1984).

[3] V. J. Clancey, "Fire hazards of calcium hypochlorite", J. Haz. Materials 1 (1976) 83-94.

[4] B. Gidas, W. M. Ni and L. Nirenberg, "Symmetry and related properties via the maximum principle", Commun. Math. Physics 68 (1979) 209-243.

[5] B. F. Gray, "Critical behaviour in chemically reacting systems I. Difficulties with the Semenov theory", Comb. Flame 21 (1993) 313-316.

[6] B. F. Gray and I. Gonda, "Effect of small-amplitude periodic parameter variation in thermokinetic systems", J. Chem. Soc. Faraday Trans. 279 (1983) 1729-1740.

[7] B. F. Gray and J. C. Jones, "Critical behaviour in layered media", Comb. Flame 40 (1981) 35-43.

[8] S. K. Scott, "Thermal explosion of dispersed media", J. Chem. Soc. Trans. Faraday 280 (1984) $1555-1568$. 
